This is postprint of the article; original publication can be found at:

https://pubs.acs.org/doi/10.1021/acssynbio.6b00353

\title{
Development of a Biosensor Concept to Detect the Production of Cluster- Specific Secondary Metabolites
}

Yi-Qian Sun ${ }^{1,2}$, Tobias Busche ${ }^{3}$, Christian Rückert ${ }^{3}$, Constanze Paulus ${ }^{4,6}$, Yuriy Rebets ${ }^{4}$, Renata Novakova ${ }^{5}$, Jörn Kalinowski ${ }^{3}$, Andriy Luzhetskyy ${ }^{4,6}$, Jan Kormanec ${ }^{5}$, Olga N. Sekurova $^{7}$, Sergey B. Zotchev ${ }^{7 *}$

${ }^{1}$ Department of Biotechnology; Norwegian University of Science and Technology, Trondheim, Norway.

${ }^{2}$ The Department of Laboratory Medicine, Children's and Women's Health (LBK), Norwegian University of Science and Technology, Trondheim, Norway.

${ }^{3}$ Center for Biotechnology, Bielefeld University, Universitätsstraße 27, 33615 Bielefeld, Germany

${ }^{4}$ Helmholtz Institute for Pharmaceutical Research Saarland, Actinobacteria Metabolic Engineering Group, Saarbrücken, Germany

${ }^{5}$ Institute of Molecular Biology, Slovak Academy of Sciences, 84551 Bratislava, Slovak Republic

${ }^{6}$ Universität des Saarlandes, Pharmaceutical Biotechnology, Saarbrücken, Germany

${ }^{7}$ Department of Pharmacognosy, University of Vienna, 1090 Vienna, Austria.

*Corresponding author: sergey.zotchev@ univie.ac.at, Tel: +43 14277 55296, Fax: +43 14277 855296. 


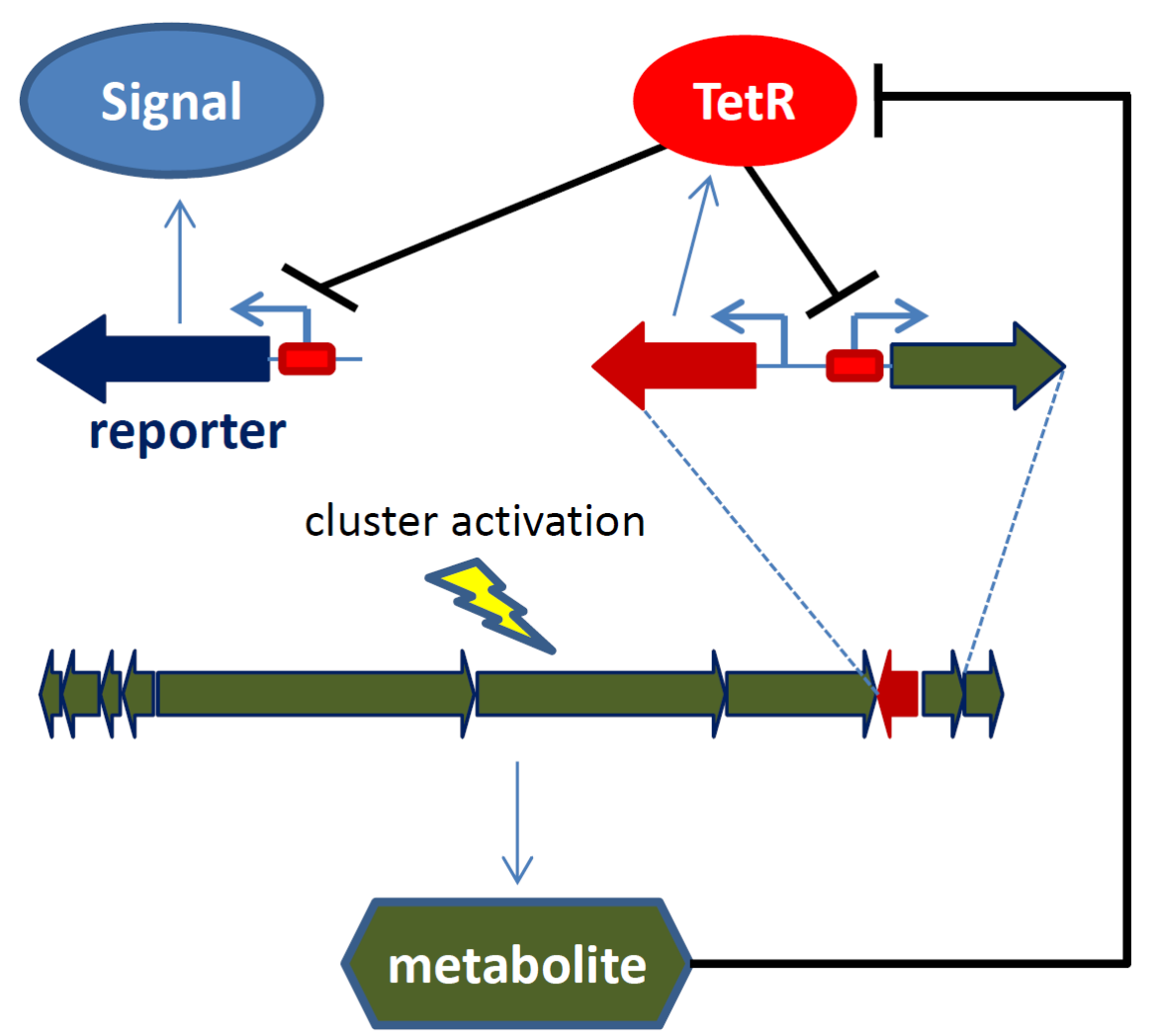

\section{ABSTRACT}

Genome mining of actinomycete bacteria aims at the discovery of novel bioactive secondary metabolites that can be developed into drugs. A new repressor-based biosensor to detect activated secondary metabolite biosynthesis gene clusters in Streptomyces was developed. Biosynthetic gene clusters for undecylprodigiosin and coelimycin in the genome of Streptomyces lividans TK24, which encoded TetR-like repressors and appeared to be almost "silent" based on the RNA-seq data were chosen for the proof-of-principle studies. The bpsA reporter gene for indigoidine synthetase was placed under control of the promotor/operator regions presumed to be controlled by the cluster-associated TetR-like repressors. While the biosensor for undecylprodigiosin turned out to be non-functional, the coelimycin biosensor was shown to perform as expected, turning on biosynthesis of indigoidine in response to the concomitant production of coelimycin. The developed reporter system concept can be applied 
to those cryptic gene clusters that encode metabolite-sensing repressors to speed up discovery of novel bioactive compounds in Streptomyces.

KEY WORDS: Streptomyces, secondary metabolite biosynthesis, orphan gene clusters, TetR repressor, biosensor

Natural products from Streptomyces and other actinomycete bacteria remain a rich source for the discovery of new bioactive compounds that may have a potential to be developed into human medicines. Recent advances in genomics of these important bacteria revealed their unprecedented capacity to synthesize structurally diverse secondary metabolites, while only a few of those are actually produced in standard laboratory conditions ${ }^{1}$. Consequently, a concept of genome mining was developed, that utilizes information on secondary metabolite biosynthesis gene clusters in order to either activate their expression in native producers, or express them heterologously in engineered bacterial hosts ${ }^{2}$. Despite some success stories ${ }^{3}$, genome mining still appears to be unpredictable in terms of outcomes and may yield unexpected results ${ }^{4}$. The most laborious part of genome mining remains analytics aimed at detection and identification of a compound expected to be produced as a result of activation or heterologous expression of a targeted gene cluster. Development of a robust biosensor that could be used to directly assess actual production of a cluster-specific compound would be of great advantage, also with respect to high-throughput screening after random mutagenesis.

Certain types of proteins provide unique opportunities for development of such biosensors. For example, resistance to tetracycline in Gram-negative bacteria is due to the export of this antibiotic by the TetA efflux pump. Expression of tetA is under tight transcriptional control of the repressor TetR, which is encoded by a divergently transcribed gene. Upon binding to tetracycline, TetR dissociates from its operator upstream of $t e t A$, thus allowing its transcription, 
and consequently, efflux of tetracycline by TetA ${ }^{5}$. The first biosensor capable of detecting oxytetracycline production by Streptomyces rimosus was constructed in Escherichia coli based on the original TetR repressor ${ }^{6}$. TetR-like repressor-coding genes have been identified within or in the vicinity of secondary metabolite clusters in many Streptomyces, which implied their potential roles in sensing cluster-specific molecules. Some of such repressors were indeed confirmed to bind both the end products of cluster-specific biosynthetic pathways, and/or its precursors. One of the first examples that concerned TetR-like repressors responding to secondary metabolites other than tetracyclines was that of LanK, the repressor controlling landomycin biosynthetic pathway in Streptomyces cyanogenus ${ }^{7}$. This protein was shown to bind various landomycins with altered aglycon structure, as well as extremely low concentration of the final product, landomycin A. Consequently, the authors have suggested that LanK can be used to develop a biosensor for detection of landomycin-related compounds. The Repressor function of SymR, a TetR-like protein encoded by the simocyclinone biosynthesis gene cluster of S. antibioticus, was shown to be abrogated upon its binding to either simocyclinone D8 or its precursor $\mathrm{C}^{8}$. Recently, chlorothricin and its biosynthetic intermediates were shown to modulate binding of a TetR-like repressor ChlF1 to its target genes in the chlorothricin gene cluster of Streptomyces antibioticus ${ }^{9}$.

These findings prompted us to investigate if TetR-like repressors in Streptomyces can be used for development of robust biosensors, which would couple easily detectable expression of the reporter with the production of specific secondary metabolites. Such biosensor would represent a clear advantage over the earlier described reporter system that detects expression of the secondary metabolite biosynthesis genes, as described by Guo et al. for gaudimycin biosynthesis. ${ }^{10}$ Indeed, our design would allow direct monitoring of the cluster-specific product, not just expression of its biosynthetic genes. Here, we present a proof-of-principle for such a 
biosensor, and argue that similar design can greatly assist in the genome-based biosprospecting for novel bioactive compounds in Streptomyces and other actinomycete bacteria.

\section{RESULTS AND DISCUSSION}

\section{Identification of Poorly Expressed Secondary Metabolite Biosynthesis Gene Clusters in}

Streptomyces lividans TK24. Analysis of the $S$. lividans TK24 genome ${ }^{11}$ using online version of the software antiSMASH $3.0^{12}$ revealed 27 gene clusters for the biosynthesis of secondary metabolites, but this number was amended to 29 after manual curation using BLAST searches (Table 1). Out of those, 17 gene clusters for known compounds were identified, governing biosynthesis of actinorhodin, undecylprodegiosin, coelibactin, albaflavenone, coelimycin, hopanoids, desferroxamine, lanthionine-containing peptide SapB, 2-methylisoborneol, calcium-dependent antibiotic, melanin, spore pigment, ectoine, coelichelin, 1,3,6,8tetrahydroxynaphthalene, isorenieratene and geosmin. It is worth noting that S. lividans TK24 has two identical gene clusters for the biosynthesis of albaflavenone, apparently located within the terminal inverted repeats of the linear chromosome. Next, we decided to explore the transcriptional landscape of the secondary metabolite biosynthesis gene clusters using RNAseq technology ${ }^{13}$. Total RNA was isolated from S. lividans TK24 cultures growing in liquid minimal media with or without addition of casamino acids at early and late logarithmic phases, as well as at stationary phase. The mRNA fractions were enriched by depletion of stable RNA and then converted to strand-specific transcriptome libraries. After sequencing by Illumina technology, gene expression analysis was performed for every scaffold-building gene of a secondary metabolite gene cluster by calculating the transcripts per million reads (TPM) in order to find transcriptionally silent and therefore putatively repressed secondary metabolite gene clusters (a detailed description of the RNA-seq experiments will be published elsewhere). 
We specifically examined the data for the gene clusters encoding TetR-like repressors, namely those for 2-methylisoborneol (cluster 2), coelimycin (cluster 10), geosmin (cluster 12), undecylprodigiosine (cluster 14), spore pigment (cluster 16), actinorhodin (cluster 18), 1,3,6,8tetrahydroxynaphthalene (cluster 23), and coelichelin (cluster 25). Full overview of the gene expression profiles for the $S$. lividans TK24 secondary metabolite biosynthesis gene clusters is presented in Supplementary information, Table S1. We have focused our examination on the genes from the targeted clusters, which encode enzymes responsible for the assembly of secondary metabolite scaffolds, reasoning that such scaffolds would represent a minimal structure that TetR repressors could bind. Out of examined gene clusters, only those for coelimycin and undecylprodigiosine fulfilled the criteria of being expressed at very low levels and encoding TetR-like repressors.

\section{Attempts on Construction of the Undecylprodegiosin Biosensor}

First, we have focused on the undecylprodigiosin biosynthetic gene cluster (red, Figure 1A), which has been characterized in quite some details ${ }^{14}$. These earlier studies, however, did not address the function of the divergently transcribed genes SLIV_09075 and SLIV_09080 located ca $9 \mathrm{~kb}$ downstream of redE. SLIV_09075 encodes a TetR-like repressor, and the SLIV_09080 gene product is similar to MMPL domain-containing transporters. Since the red cluster does not encode any transporters, it seemed logical to suggest that the SLIV_09075+SLIV_09080 pair is responsible for undecylprodigiosin efflux in response to the production of this compound, with the TetR-like repressor being the receiver of the signal. Considering this, we PCR-amplified the putative promoter/operator region upstream of SLIV_09080, and placed it upstream of the $b p s A$ reporter in an integrative plasmid. BpsA is a mono-modular non-ribosomal peptide synthetase responsible for the biosynthesis of the blue pigment indigoidine, which is easily detectable both visually and spectroscopically ${ }^{15}$. When 
the resulting putative undecylprodigiosin reporter plasmid pYQS042 (Table S1) was introduced into S. lividans TK24, no indigoidine production was observed in the recombinant strain, suggesting that the SLIV_09075-encoded repressor blocks expression of bpsA.

Protein RedD encoded by the SLIV_09220 gene in the red cluster of S. lividans was shown to be a positive activator of the undecylprodigiosin gene cluster in $S$. coelicolor ${ }^{16}$. Therefore, overexpression of RedD was expected to trigger biosynthesis of undecylprodigiosin in $S$. lividans as well, to which the constructed biosensor would react. Consequently, genes encoding RedD (SLIV_09220) and another putative regulator of the LuxR family, RedZ (SLIV_09200) ${ }^{14}$ in the red cluster were overexpressed in the recombinant S. lividans TK24 harboring reporter plasmid pYQS042. Overexpression of both redD and redZ led to apparent activation of undecylprodigiosin production (activation was more efficient upon overexpression of redD), while no production of indigoidine could be observed in the engineered strains. These results implied that the TetR-like repressor encoded by SLIV_09075 does not bind undecylprodigiosin, and its gene may not belong to the red cluster.

In the next attempt to construct undecylprodigiosin-specific biosensor, we decided to combine the features of TetR-like repressor and LuxR-type activator in one hybrid protein. The Nterminal part of LuxR activator proteins are known to contain autoinducer-binding domains that have affinity to $\mathrm{N}$-acyl homoserine lactones (AHLs) ${ }^{17}$. Taking into account the fact that no AHL synthase is encoded by the red cluster, and recent reports on the LuxR "solos" that respond to diverse small molecules instead of $\mathrm{AHLs}^{18}$, we considered it possible that an Nterminal domain in the RedZ response regulator may sense undecylprodigiosin or its precursors. Therefore, we decided to generate hybrid proteins that would combine the DNA binding domain of the TetR-like repressor encoded by SLIV_09075 and a putative metabolite-sensing domain of RedZ. The rationale behind this attempt to construct hybrid repressors stems from the earlier reported success in creating a hybrid transcription factor that combines the 
regulatory and activation domains of cAMP-responsive element binding protein with the DNA binding domain of the TetR repressor ${ }^{19}$. Functional metabolite-sensing hybrid repressors have also been reporter by Keasling group, which combined domains of metabolic enzymes to the DNA-binding domain of $\operatorname{TetR}^{20}$. Consequently, we constructed two versions of a hybrid repressor that, providing our assumption noted above was correct, could respond to the production of undecylprodigiosin. These versions were made by linking a putative $\mathrm{N}$-terminal receiver domain of RedZ with the helix-turn-helix (HTH) DNA binding domain of the SLIV_09075-encoded TetR-like repressor. The domain boundaries of both proteins were predicted using online ProDom software tool, corresponding parts of the genes were amplified by PCR from the genomic DNA of S. lividans TK64 and fused using Gibson ligation (see Methods section for details).

The two versions of hybrid repressor were made with different inter-domain linkers originating either from RedZ (for HR1 version) or TetR-like (for HR2 version) regulators (Figure 1B, Table S1: constructs pYQS065 and pYQS066, respectively). The wild type copy of the SLIV_09075 gene was replaced by either of the SLIV_09075-redZ hybrid genes in S. lividans TK24 using double crossover recombination. The reporter construct pYQS042 was then integrated into the chromosomes of the strains carrying hybrid genes. Both resulting recombinant strains showed production of indigoidine without overexpression of redD, suggesting that the hybrid proteins lost the repressor function characteristic of SLIV_09075. Most likely, the HTH DNA-binding domain of SLIV_09075 in hybrid proteins could no longer bind to the operator upstream of the reporter gene, probably due to the perturbations in their tertiary structures. Yet another possible explanation could be that the hybrid proteins acquired an activator function and, in the absence of the intact SLIV_09075, can activate expression of the $\operatorname{bps} A$ reporter gene. In order to simplify visual detection of indigoidine production, which could be masked by actinorhodin and undcylprodigiosin, a recombinant strain S. lividans 
RedStrep1 was constructed, which lacks red and act clusters, but retains SLIV_09075. To delete both clusters in S. lividans TK24, an efficient deletion system based on the positive selection of double crossover events with $b p s A$ reporter gene was used ${ }^{14}$ (see Methods for details). Two RedStrep1-based recombinant strains were constructed, which harbored both the integrated reporter pYQS042, and a low-copy number autonomously replicating plasmid expressing HR1 or HR2 proteins under control of the $e r m E^{*}$ p promoter (Table S2). No indigoidine production was observed by these recombinant strains, which excluded the possibility that hybrid proteins had direct activator function and could outcompete the SLIV_09075-encoded repressor. In summary, hybrid proteins constructed here proved to be non-functional, prompting further studies toward robust design of the hybrid TetR-like repressors.

Construction of the Coelimycin-Specific Biosensor. Our failure to construct undecylprodigiosin-specific biosensor was mainly due to the fact that TetR-like repressor encoding gene located at the border of the red cluster apparently had no function in the biosynthesis of this antibiotic. To establish a proof of concept, we next focused on the coelimycin gene cluster ( $c p k$ ) for biosensor development, since this compound has never before been identified in S. lividans, while methods for its detection were available from the earlier reports on the closely related strain $S$. coelicolor M145. ${ }^{21}$. Coelimycin is a polyketide alkaloid, the biosynthetic gene cluster for which in $S$. coelicolor is silent, but can be activated upon deletion of the $s c b R 2$ repressor gene ${ }^{22}$. In $S$. coelicolor, $s c b R 2$ is located proximal (but transcribed in opposite direction) to the gene scoT encoding a type II thioesterase that proved to be important for coelimycin biosynthesis in the latter organism ${ }^{23}$. In S. lividans TK24, the arrangement of genes is similar (Figure 2), and it was safe to assume that the SLIV_06715encoded repressor would control the expression of the divergently transcribed thioesterase II- 
encoding gene SLIV_06710. We PCR-amplified putative promoter/operator region upstream of SLIV_06710, and placed the bpsA reporter gene under its control in an integrative vector pSET152 $2^{24}$. This reporter construct pYQS040 was integrated into the chromosome of $S$. lividans RedStrep1. The reporter strain RedStrep1 [pYQS040] showed no indigoidine production in a range of conditions, indicating that the SLIV_06715-encoding repressor acts in trans, and blocks the transcription of the reporter gene.

\section{Testing of the Coelimycin Biosensor Using Artificial Activation of the cpk Gene Cluster.}

In order to obtain proof-of-principle for our biosensor system, we decided to force coelimycin production in the reporter strain RedStrep1 [pYQS040] using overexpression of a pathwayspecific regulator. The latter strategy was shown to be useful in genome mining aimed at the discovery of novel compounds ${ }^{25}$. However, this approach may lead to unexpected results, and detection of the products of potentially activated clusters may prove difficult, especially in case of complex and unusual clusters ${ }^{4}$. The $c p k$ gene cluster in S. lividans encodes two putative positive regulators of the SARP family, SLIV_06705 (CpkN) and SLIV_06745 (CpkO) (Fig. 1). The $c p k O$ gene product was previously shown in $S$. coelicolor to positively regulate the $c p k$ gene cluster expression ${ }^{26}$, while the function of $c p k N$ gene was unclear. These putative regulatory genes were expressed under control of the PermE promoter from the SCP2*-based replicative plasmids pYQS060 ( $c p k N)$ and pYQS061 (cpkO) in strain RedStrep1 [pYQS040]. Indigoidine production was observed in both RedStrep1 [pYQS040/pYQS060] and RedStrep1 [pYQS040/pYQS061] recombinant strains, which indicated activation of the $c p k$ cluster. The recombinant strain overexpressing SLIV_06705 also showed more pronounced indigoidine production, suggesting that this SARP regulator is a stronger activator for the $c p k$ cluster. In order to confirm that positive regulator-induced reporter expression was due to the relief of the SLIV_06715-encoded repressor upon coelimycin binding, gene SLIV_06770 encoding a 
scaffold-building PKS in the $c p k$ cluster was inactivated by insertion of a suicide vector pYQS070. The resulting recombinant colonies of RedStrep1 [pYQS040/pYQS060/pYQS070] were no longer producing indigoidine, thus confirming that the developed biosensor system indeed responds to the production of coelimycin (Fig. 2).

To obtain a final proof of coelimycin production, we compared metabolic profiles of the recombinant strains S. lividans RedStrep1 [pYQS040] carrying just the biosensor, and RedStrep1 [pYQS040/pYQS060] that in addition expressed the SARP activator RedD. The recombinant strains were cultivated in several commonly used liquid media (TSB, SG, NL19, phage medium and R5A) and extracted metabolites were analyzed by LC-MS. When grown in R5A or SG media strain RedStrep1 [pYQS040/pYQS060] was found to produce both coelimycin P1 and blue pigment indigoidine. Coelimycin P1 was by its absorption spectrum (characteristic peak at $360 \mathrm{~nm}$ ), its exact mass (detected m/z $349.1213[\mathrm{M}+\mathrm{H}]^{+}$, calculated 349.1215 $[\mathrm{M}+\mathrm{H}]^{+}$), and by its MS/MS fragmentation pattern (Fig. S2). Interestingly, trace amounts of coelimycin P1 were also detected in the extract of S. lividans RedStrep1 [pYQS040] strain when incubated in the R5A media, but not in SG. In these conditions, the biosensor did not react to the production of minute amounts of coelimycin P1, probably due to its efficient and rapid efflux from the cells. The accumulation of coelimycin P1 was not detected when strains were grown either in TSB, phage media or NL19 media. Under these conditions production of indigoidine by S. lividans RedStrep1 [pYQS040] was also not observed.

\section{CONCLUSIONS}

The genome mining approach has shown a considerable potential in discovery of novel microbial secondary metabolites ${ }^{25}$. Still, the metabolomes of prolific secondary metabolite producers are often very complex, precluding identification of cluster-specified compounds. 
Biosensors coupled to the actual production of target compounds would be very helpful in streamlining the discovery process, eliminating non-producers from the screening. In a recent report Guo et al. constructed a reporter system linking the expression of genes for gaudimycin biosynthesis to a reporter system in Streptomyces ${ }^{10}$. However, authors have reported high percentage of false-positives which, while expressing a reporter, did not actually produced a target metabolite. Also, in some cases expression of biosynthetic genes does not correlate with the compound production, probably due to some obstacles affecting translation or posttranslational modification of biosynthetic enzymes ${ }^{27}$. In our study, TetR-like repressors and bpsA reporter gene were used to construct biosensors that would directly detect production of secondary metabolites. Apparently, success in construction of a functional biosensor depends on whether identified TetR-like repressor gene actually belongs to the biosynthetic cluster, and can bind the cluster-specified compound. In the case of coelimycin, we have obtained a proofof-principle, demonstrating the utility of such a biosensor, although we cannot exclude the possibility that biosensor may also react to some intermediates in the biosynthetic pathway. Publicly available genome sequences, especially those for actinomycete bacteria, provide access to hundreds of biosynthetic gene clusters encoding TetR-like repressors, for which biosynthetic products remain unknown. Moreover, there is a plethora of other regulator families, in which the binding of a metabolite leads to the release of the repressor from its operator (e.g. LacI family regulators $)^{28}$, which are associated with the secondary metabolite biosynthesis gene clusters. Using the metabolite-sensing repressor-based biosensor principle, it will become possible to assess functional expression of these clusters either after random mutagenesis, under various cultivation conditions, or through genetic manipulation. Based on the promising results reported by Chou and Keasling on metabolite-sensing hybrid repressors ${ }^{20}$, it seems plausible that a more versatile and modular biosensor system can be developed. Indeed, fusion of a metabolite sensor represented by a domain of a biosynthetic enzyme predicted to 
bind the final product (or a critical intermediate) to a standardized part representing HTH domain that binds specific operator controlling a reporter can be done quickly and efficiently. Replacement of the receiver domain in a standardized hybrid repressor with a domain of a biosynthetic enzyme predicted to bind the final product (or a critical intermediate) would only require minimal engineering in order to tune the biosensor to a particular gene cluster.

\section{METHODS}

Bacterial Strains, Plasmids, and Growth Conditions. Description of plasmids and bacterial strains used or generated during this study is provided in Tables S1 and S2 (Supporting Information), respectively. S. lividans TK24 was used as the wild-type strain. RedStrep 1 ( $\triangle a c t$ $\Delta r e d)$ was constructed as shown on Figure S1 (Supporting Information)with the deletions of nt 2961047-2982325 (act cluster) and nt 2081767-2113444 (red cluster) in S. lividans TK24 genome $^{11}$. The deletions were, were validated by genome sequencing. RedStrep 1 was used in this study to avoid the potential color disturbance due to production of pigmented antibiotics specified by the act and red clusters in S. lividans TK24.

Escherichia coli strains were grown in Luria-Bertani (LB) broth or on LB agar. XL1-blue was used for general cloning. ET12567 (pUZ8002) was used for intergeneric transfer of plasmids to Streptomyces with the standard procedure of conjugation ${ }^{28}$. Streptomyces spore preparation was obtained by growing on soy flour mannitol medium (SFM) at $30^{\circ} \mathrm{C}$ for $4-7$ days. Antibiotics were supplemented to growth medium at the following concentrations when applicable: ampicillin $100 \mu \mathrm{g} / \mathrm{ml}$; apramycin 50 or $100 \mu \mathrm{g} / \mathrm{ml}$; chloramphenicol $20 \mu \mathrm{g} / \mathrm{ml}$; hygromycin $100 \mu \mathrm{g} / \mathrm{ml}$; kanamycin $25 \mu \mathrm{g} / \mathrm{ml}$; nalidixic acid $30 \mu \mathrm{g} / \mathrm{ml}$, and thiostrepton 30 $\mu \mathrm{g} / \mathrm{ml}$. 
RNA isolation, library preparation and cDNA sequencing. S. lividans TK24 cells were grown in MM media supplemented with and without casamino acids. Samples were taken at early and late logarithmic phases, as well as at stationary phase. Harvesting and RNA isolation were performed as previously described [https://www.ncbi.nlm.nih.gov/pubmed/22943411]. RNA quality and quantity was checked by an Agilent 2100 Bioanalyzer (Agilent Technologies, Böblingen, Germany) and Trinean Xpose system (Gentbrugge, Belgium) prior and after rRNA depletion by using the Ribo-Zero rRNA Removal Kit (Bacteria) (Illumina, San Diego, CA, USA). The TruSeq Stranded mRNA Library Prep Kit (Illumina, San Diego, CA, USA) was used to prepare the cDNA libraries, which were then sequenced in paired-end mode on an Illumina HiSeq 1500 system (San Diego, CA, USA) with 2 x 27 nt read length.

\section{Read mapping, data visualization and analysis of gene expression.}

Reads were trimmed to a minimum of 20 bases in length using Trimmomatic V0.33 ${ }^{29}$. Mapping of the trimmed reads to the S. lividans TK24 genome sequence ${ }^{10}$ was performed with Bowtie 2 using standard settings ${ }^{30}$. ReadXplorer 2.2.0 $0^{31}$ was used for visualization of short read alignments and calculation of transcripts per million reads (TPM) of every scaffold-building gene of a secondary metabolite gene cluster ${ }^{32}$.

DNA Manipulation. General DNA cloning, DNA digestion with restriction enzymes and plasmid transformation into Escherichia coli were performed as described in Sambrook et al. ${ }^{33}$. PCR fragments were amplified using MasterAmp ${ }^{\mathrm{TM}}$ Extra-Long DNA Polymerase Mix and buffers (Epicentre, USA). The primers used in this study were described in Table S1. Primers for PCR were designed using Clone Manager Professional Version 9 (Sci-Ed Software, USA). To construct new vectors, PCR and vector fragments were assembled either by T4 DNA ligase or Gibson ligation ${ }^{34}$. Recombinant constructs were verified by DNA sequencing by GATC 
Biotech. Streptomyces genomic DNA was isolated using Wizard ${ }^{\circledR}$ Genomic DNA Purification Kit (Promega, USA).

Construction of $S$. lividans RedStrep1 strain. Two DNA fragments flanking the act cluster were PCR amplified from the genomic DNA of S. lividans TK24. A 2.7-kb DNA fragment flanking the 3' end of SLIV_12925 (Fig. S1A) was amplified with the primers 12925Dir and 12925Rev (Table S4). This DNA fragment was digested using $\mathrm{MfeI}$ and ClaI and cloned into pAMR $4{ }^{15}$ resulting in pAct1. A 3-kb DNA fragment flanking the 3' end of SLIV_13030 (Fig. S1) was amplified with the primers 13030Dir and 130130Rev (Table S4). This DNA fragment was digested with AflII and HindIII and cloned in pAcl1, resulting in pAct2. The plasmid was introduced into $S$. lividans TK24 by conjugation, and after several rounds of propagation bpsAnegative apramycin-resistant and kanamycin-sensitive colonies (indicating a double cross-over event) were selected. The replacement of the act cluster by apramycin-resistance cassette was confirmed by Southern-blot hybridization (data not shown). One clone (S. lividans $\Delta a c t$ ) was used for further deletion of the red cluster. Likewise, two DNA fragments flanking the red cluster were PCR amplified from the genomic DNA of S. lividans TK24. A 2.9-kb DNA fragment flanking the 5' end of SLIV_09220 (Fig. S1A) was amplified with the primers 9220Dir and 9220Rev (Table S4), digested with $M f e I$ and BamHI and cloned into pSPR11, resulting in pRed1. The cloning vector pSPR11 was prepared by replacing the apramycin-resistance gene aac(3)IV in pAMR4 with synthetic spectinomycin-resistance gene aadA from the plasmid paatsaadA-oriT, containing oriT and $P-G G$ and $G-C C$ recombination sites for phiC31 recombinase $^{35}$. A 3-kb DNA fragment flanking the 3' end of SLIV_13030 (Fig. S1A) was amplified with the primers 9115Dir and 9115Rev (Table S4), digested with AflII and HindIII and cloned in $\mathrm{pRed} 1$ resulting in $\mathrm{pRed} 2$. The plasmid was introduced into $S$. lividans $\Delta$ act by conjugation with selection for spectinomycin resistance. After several rounds of sporulation, 
four bpsA-negative spectinomycin-resistant and kanamycin-sensitive colonies were selected, and the correct double crossover event in the red cluster was confirmed by Southern-blot hybridization (data not shown). One $S$. lividans $\Delta$ act $\Delta$ red clone was used for further studies. Both antibiotic resistance markers, the apramycin resistance marker with FRT sites for recombination ${ }^{36}$, and the spectinomycin resistance marker with $B-C C$ and $P-G G$ sites for recombination, were removed from the $S$. lividans $\Delta a c t \Delta$ red strain by successive transformation of the plasmid pUWLHFLP, containing Flp recombinase ${ }^{37}$ and pKHint31, containing phiC31 recombinase $\mathrm{s}^{38}$. The removal of both markers was confirmed by antibiotic sensitivity tests after five rounds of nonselective sporulation. Elimination of the plasmids carrying recombinase genes was achieved after two rounds of nonselective propagation. The representative clone from the resulting strain, S. lividans $\triangle a c t-F R T \Delta r e d-B C C$, later termed RedStrep1, was verified by Southern blot hybridization (Fig. S1B) using probes for both clusters.

Construction of genes encoding hybrid proteins. The products of genes SLIV_09075 and SLIV_09200 (redZ) were analysed using ProDom online tool (http://prodom.prabi.fr/prodom/current/ html/home.php) in order to predict protein domain boundaries. Accordingly, hybrid proteins of the following compositions were designed: (i) HR1 would contain C-terminal HTH domain of the SLIV_09075 gene product with a Cterminal linker (aa 1-104) fused to the putative receiver domain of RedZ (aa 1-115); (HR2 would contain C-terminal HTH domain of the SLIV_09075 gene product (aa 1-78) fused to the putative receiver domain of RedZ followed by its C-terminal linker (aa 1-145). Parts of the corresponding genes encoding respective domains were amplified by PCR from the genomic DNA of S. lividans TK24 (primers given in Table S3, Supporting Information) and joined using 
Gibson ligation. ${ }^{35}$ The resulting fragments were cloned into the $\mathrm{pKC} 1218 \mathrm{HygR}$ vector under control of $e r m E^{*}$ p promoter, and verified by DNA sequencing.

Analysis of Secondary Metabolites produced by the Recombinant Strains. Strains were grown for 3 days at $28{ }^{\circ} \mathrm{C}$ in $10 \mathrm{ml}$ of TSB medium. Subsequently, $4 \mathrm{ml}$ of the pre-culture was used to inoculate $50 \mathrm{ml}$ of production media in $500 \mathrm{ml}$ flasks: NL19, SG, R5A or phage medium. The main cultures were cultivated at $28^{\circ} \mathrm{C}$ on a rotary shaker at $180 \mathrm{rpm}$ for 5 days. Metabolites were extracted with acetone:methanol 1:1 from biomass and with ethyl acetate from cultural liquid, solvents were evaporated and extracts were dissolved in $300 \mu \mathrm{l}$ of methanol.

Samples were separated on a Dionex Ultimate 3000 RSLC system (Thermoscientific ) using a BEH C18, $100 \times 2.1 \mathrm{~mm}, 1.7 \mu \mathrm{m} \mathrm{d}_{\mathrm{p}}$ column (Waters) with linear gradient of solvent $\mathrm{B}$ (acetonitrile with $0.1 \%$ of formic acid) against solvent A (water with $0.1 \%$ of formic acid) at a flow rate of $600 \mu \mathrm{l} / \mathrm{min}$ and $45^{\circ} \mathrm{C}$. The gradient started by a $0.5 \mathrm{~min}$ isocratic step at $5 \% \mathrm{~B}$ then increased to $95 \%$ B over 9 min to end up with a 2 min isocratic step at $95 \%$ B before reequilibration under the initial conditions. UV spectra were acquired by a DAD in the range of 200 to $600 \mathrm{~nm}$. The mass-spec data was collected on an amaZON speed resolution mass spectrometer (Bruker Daltonics, Germany) using ESI source. Mass spectra were acquired in centroid mode ranging from 100 to $2000 \mathrm{~m} / \mathrm{z}$ at a $2 \mathrm{~Hz}$ scan rate. Data was collected and analysed with the Bruker Compass Data Analysis software, version 4.2 (Bruker, Billerica, USA).

\section{ASSOCIATED CONTENT}

Supporting Information: Figure S1 (construction of S. lividans RedStrep1 strain), Figure S2 (identification of coelimycin P1), Tables S1-S4 (descriptions of bacterial strains, plasmids, oligonucleotides, supporting main text). 


\section{AUTHOR INFORMATION}

Corresponding Author

*E-mail: sergey.zotchev@univie.ac.at

\section{Notes}

The authors declare no competing financial interest.

\section{ACKNOWLEDGMENTS}

This work was supported by the European Commission under the $7^{\text {th }}$ Framework Program through the "Collaborative Project" action "STREPSYNTH” Grant No. 613877, the University of Vienna and the Slovak Research and Development Agency under the contract No. DO7RP0037-12. JK was also supported by the Slovak Research and Development Agency under the contract APVV-15-0410. We are thankful to Kristel Bernaerts and Wouter Daniels (KU Leuven, Belgium) for growing S. lividans TK24 for the RNA-seq experiments.

\section{REFERENCES}

(1) Hopwood, D.A. (2006) Soil to genomics: the Streptomyces chromosome. Annu. Rev. Genet. 40, 1-23.

(2) Zarins-Tutt, J.S., Barberi, T.T., Gao, H., Mearns-Spragg, A., Zhang, L., Newman, D.J., and Goss, R.J. (2016) Prospecting for new bacterial metabolites: a glossary of approaches for inducing, activating and upregulating the biosynthesis of bacterial cryptic or silent natural products. Nat. Prod. Rep. 33, 54-72.

(3) Katz, L., and Baltz, R.H. (2016) Natural product discovery: past, present, and future. $J$. Ind. Microbiol. Biotechnol. 43, 155-176. 
(4) Sekurova, O.N., Pérez-Victoria, I., Martín, J., Degnes, K.F., Sletta, H., Reyes, F., and Zotchev, S.B. (2016) New Deferoxamine Glycoconjugates Produced upon Overexpression of Pathway-Specific Regulatory Gene in the Marine Sponge-Derived Streptomyces albus PVA9407. Molecules 21, pii: E1131.

(5) Cuthbertson, L., and Nodwell, J. R. (2013) The TetR family of regulators, Microbiol. Mol. Biol. Rev. 77, 440-475.

(6) Hansen, L.H., Ferrari, B., Sørensen, A.H., Veal, D., and Sørensen, S.J. (2001) Detection of oxytetracycline production by Streptomyces rimosus in soil microcosms by combining whole-cell biosensors and flow cytometry. Appl. Environ. Microbiol. 67, 239-244.

(7) Ostash, B., Ostash, I., Zhu, L., Kharel, M.K., Luzhetskiı̌, A., Bechthold, A., Walker, S., Rohr, J., and Fedorenko, V. (2010) Properties of lanK-based regulatory circuit involved in landomycin biosynthesis in Streptomyces cyanogenus S136. Genetika 46, 604-609.

(8) Le, T.B., Fiedler, H.P., den Hengst, C.D., Ahn, S.K., Maxwell, A., and Buttner, M.J. (2009) Coupling of the biosynthesis and export of the DNA gyrase inhibitor simocyclinone in Streptomyces antibioticus. Mol. Microbiol. 72, 1462-1474.

(9) Li, Y., Li, J., Tian, Z., Xu, Y., Zhang, J., Liu, W., and Tan, H. (2016) Coordinative Modulation of chlorothricin biosynthesis by binding of the glycosylated intermediates and end product to a responsive regulator ChlF1. J. Biol. Chem. 291, 5406-5417.

(10) Guo, F., Xiang, S., Li, L., Wang, B., Rajasarkka, J., Grondahl-Yli-Hannuksela, K., Ai, G., Metsa-Ketela, M., and Yang, K. (2015) Targeted activation of silent natural product biosynthesis pathways by reporter-guided mutant selection. Metab. Eng. 28, 134-142.

(11) Rückert, C., Albersmeier, A., Busche, T., Jaenicke, S., Winkler, A., Friethjonsson, O. H., Hreggviethsson, G. O., Lambert, C., Badcock, D., Bernaerts, K., Anne, J., Economou, A., and Kalinowski, J. (2015) Complete genome sequence of Streptomyces lividans TK24, J. Biotechnol. 199, 21-22. 
(12) Weber, T., Blin, K., Duddela, S., Krug, D., Kim, H. U., Bruccoleri, R., Lee, S. Y., Fischbach, M. A., Muller, R., Wohlleben, W., Breitling, R., Takano, E., and Medema, M. H. (2015) antiSMASH 3.0-a comprehensive resource for the genome mining of biosynthetic gene clusters, Nucleic Acids Res. 43, W237-W243.

(13) Creecy, J.P., and Conway, T. (2015) Quantitative bacterial transcriptomics with RNAseq. Curr. Opin. Microbiol. 23, 133-140.

(14) Williamson, N. R., Fineran, P. C., Leeper, F. J., and Salmond, G. P. (2006) The biosynthesis and regulation of bacterial prodiginines. Nat. Rev. Microbiol. 4, 887-899.

(15) Knirschova, R., Novakova, R., Mingyar, E., Bekeova, C., Homerova, D., and Kormanec, J. (2015) Utilization of a reporter system based on the blue pigment indigoidine biosynthetic gene bpsA for detection of promoter activity and deletion of genes in Streptomyces. J. Microbiol. Methods. 113, 1-3.

(16) Takano, E., Gramajo, H. C., Strauch, E., Andres, N., White, J., and Bibb, M. J. (1992) Transcriptional regulation of the redD transcriptional activator gene accounts for growthphase-dependent production of the antibiotic undecylprodigiosin in Streptomyces coelicolor A3(2), Mol Microbiol 6, 2797-2804.

(17) Chen, J., and Xie, J. (2011) Role and regulation of bacterial LuxR-like regulators. J. Cell Biochem. 112, 2694-2702.

(18) Patel, H.K., Suárez-Moreno, Z.R., Degrassi, G., Subramoni, S., González, J.F., and Venturi, V. (2013) Bacterial LuxR solos have evolved to respond to different molecules including signals from plants. Front. Plant Sci. 4, 447.

(19) Keeley, M.B., Busch, J., Singh, R., and Abel, T. (2005) TetR hybrid transcription factors report cell signaling and are inhibited by doxycycline. Biotechniques 39, 529-536.

(20) Chou, H.H., and Keasling, J.D. (2013) Programming adaptive control to evolve increased metabolite production. Nat. Commun. 4, 2595. 
(21) Gomez-Escribano, J.P., Song, L., Fox, D.J., Yeo, V., Bibb, M.J., and Challis, G.L. (2012) Structure and biosynthesis of the unusual polyketide alkaloid coelimycin P1, a metabolic product of the cpk gene cluster of Streptomyces coelicolor M145. Chem. Sci. 3, 2716-2720.

(22) Gottelt, M., Kol, S., Gomez-Escribano, J.P., Bibb, M., and Takano, E. (2010) Deletion of a regulatory gene within the $c p k$ gene cluster reveals novel antibacterial activity in Streptomyces coelicolor A3(2). Microbiology 156, 2343-2353.

(23) Kotowska, M., Ciekot, J., and Pawlik, K. (2014) Type II thioesterase ScoT is required for coelimycin production by the modular polyketide synthase Cpk of Streptomyces coelicolor A3(2). Acta Biochim. Pol. 61, 141-147.

(24) Bierman, M., Logan, R., O’Brien, K., Seno, E.T., Rao, R.N. \& Schoner, B.E. (1992) Plasmid cloning vectors for the conjugal transfer of DNA from Escherichia coli to Streptomyces spp. Gene 116, 43-49.

(25) Rutledge, P. J., and Challis, G. L. (2015) Discovery of microbial natural products by activation of silent biosynthetic gene clusters, Nat. Rev. Microbiol. 13, 509-523.

(26) Takano, E., Kinoshita, H., Mersinias, V., Bucca, G., Hotchkiss, G., Nihira, T., Smith, C.P., Bibb, M., Wohlleben, W., and Chater, K. (2005) A bacterial hormone (the SCB1) directly controls the expression of a pathway-specific regulatory gene in the cryptic type I polyketide biosynthetic gene cluster of Streptomyces coelicolor. Mol. Microbiol. 56, 465-479.

(27) Engelhardt, K., Degnes, K.F., and Zotchev, S.B. (2010) Isolation and characterization of the gene cluster for biosynthesis of the thiopeptide antibiotic TP-1161. Appl. Environ. Microbiol. 76, 7093-7101.

(28) Swint-Kruse, L., and Matthews, K.S. (2009) Allostery in the LacI/GalR family: variations on a theme. Curr. Opin. Microbiol. 12, 129-137.

(29) Kieser, T., Bibb, M.J., Buttner, M.J., Chater, K.F., and Hopwood, D.A. (2000) Practical Streptomyces Genetics, John Innes Foundation, Norwich. 
(30) Bolger, A.M., Lohse, M., and Usadel, B. (2014) Trimmomatic: a flexible trimmer for Illumina sequence data. Bioinformatics 30, 2114-2120.

(31) Langmead, B., and Salzberg, S.L. (2012) Fast gapped-read alignment with Bowtie 2. Nat. Methods 9, 357-359.

(32) Hilker, R., Stadermann, K.B., Doppmeier, D., Kalinowski, J., Stoye, J., Straube, J., Winnebald, J., and Goesmann, A. (2014) ReadXplorer--visualization and analysis of mapped sequences. Bioinformatics 30, 2247-2254.

(33) Wagner, G.P., Kin, K., and Lynch, V.J. (2012) Measurement of mRNA abundance using RNA-seq data: RPKM measure is inconsistent among samples. Theory Biosci. 131, 281285.

(34) Sambrook, J., Fritsch, E., and Maniatis, T. (1989) Molecular cloning: a laboratory manual. 2nd ed. Cold Spring Harbor: Cold Spring Harbor Laboratory Press.

(35) Gibson, D. G., Young, L., Chuang, R. Y., Venter, J. C., Hutchison, C. A., 3rd, and Smith, H. O. (2009) Enzymatic assembly of DNA molecules up to several hundred kilobases, Nat. Methods 6, 343-345.

(36) Myronovskyi, M., Rosenkranzer, B., and Luzhetskyy, A. (2014) Iterative marker excision system. Appl. Microbiol. Biotechnol. 98, 4557-4570.

(37) Gust, B., Challis, G.L., Fowler, K., Kieser, T., and Chater, K.F. (2003) PCR-targeted Streptomyces gene replacement identifies a protein domain needed for biosynthesis of the sesquiterpene soil odor geosmin. Proc. Natl. Acad. Sci. USA 18, 1541-1548.

(38) Fedoryshyn, M., Petzke, L., Welle, E., Bechthold, A., and Luzhetskyy, A. (2008) Marker removal from actinomycetes genome using Flp recombinase. Gene 419, 43-47. 
Table 1. Secondary metabolite biosynthesis gene clusters in the genome of S. lividans TK24 identified using antiSMASH 3.0, followed by manual curation. Clusters misannotated or missed by the software via artificial fusion of several clusters are marked with *.

\begin{tabular}{|c|c|c|c|c|}
\hline No & Cluster type & $\begin{array}{l}\text { TetR-like } \\
\text { repressor }\end{array}$ & Product & $\begin{array}{l}\text { Scaffold-building gene } \\
\text { expression, max TPM* }\end{array}$ \\
\hline 1 & Terpene & - & Terpene & SLIV_00150 (1.7) \\
\hline $2 *$ & Terpene & + & 2-methylisoborneol & SLIV_00800 (324.1) \\
\hline 3 & NRPS & + & Coelibactin & SLIV_00885 (179.9) \\
\hline $4 *$ & PKSIII & - & Polyketide & SLIV_00945 (0.28) \\
\hline 5 & Indole & - & Indole (putative) & SLIV_01965 (16.0) \\
\hline $6^{*}$ & Other & - & Unknown, likely false-positive & N/A \\
\hline 7 & Terpene & - & Hopanoids & SLIV_04695 (109.3) \\
\hline 8 & Lantipeptide & - & Lanthionone-containing peptide SapB & SLIV_05105 (35.4) \\
\hline 9 & NRPS & - & Non-ribosomally synthesized dipeptide & SLIV_06265 (20.7) \\
\hline 10 & PKS I-butyrolactone & + & Coelimycin & SLIV_06770 (0.4) \\
\hline 11 & Siderophore & - & Siderophore (putative) & SLIV_07005 (4.1) \\
\hline $12^{*}$ & Terpene & + & Geosmin & SLIV_07775 (417.9) \\
\hline 13 & Bacteriocin & - & Bacteriocin (putative) & SLIV_07915 (955.7) \\
\hline
\end{tabular}




\begin{tabular}{|c|c|c|c|c|}
\hline 14 & PKS I & + & $\underline{\text { Undecylprodigiosin }}$ & SLIV_09145 (2.0) \\
\hline 15 & Siderophore & - & Siderophore (putative) & SLIV_09615 (7.5) \\
\hline 16 & PKS II & - & $\underline{\text { Spore pigment }}$ & SLIV_11780 (5.9) \\
\hline 17 & Terpene & - & $\underline{\text { Albaflavenone }}$ & SLIV_12950 (192.9) \\
\hline 18 & PKSII & + & $\underline{\text { Actinorhodin }}$ & SLIV_21540 (3.4) \\
\hline 19 & NRPS & - & $\underline{\text { Calcium-dependent antibiotic }}$ & SLIV_23760 (1035.8) \\
\hline 20 & Siderophore & - & $\underline{\text { Desferroxamine B }}$ & SLIV_24170 (19.8) \\
\hline 21 & Melanin & - & $\underline{\text { Melanin }}$ & SLIV_28385 (1011.2) \\
\hline 22 & Ectoine & - & $\underline{\text { Ectoine }}$ & SLIV_31765 (27.7) \\
\hline $23 *$ & PKS III & + & $\underline{1,3,6,8-t e t r a h y d r o x y n a p h t h a l e n e}$ & SLIV_34165 (71.5) \\
\hline 24 & Bacteriocin & - & Bacteriocin (putative) & SLIV_35480 (217.1) \\
\hline 25 & NRPS & + & $\underline{\text { Coelichelin }}$ & SLIV_36570 (38.7) \\
\hline 26 & Lantipeptide & - & Class I lantipeptide (putative) & SLIV_36960 (32.3) \\
\hline 27 & Terpene & - & $\underline{\text { Isorenieratene }}$ & TLIV_37285 (10.6) \\
\hline $28 *$ & PKS I & - & Polyunsaturated fatty acid (putative) & $37475(1.8)$ \\
\hline & Terpene & - & - & \\
\hline
\end{tabular}


Figure 1. (A) Organization of the undecylprodegiosin biosynthesis gene cluster and its right flank in S. lividans TK24. Regulatory genes redD and redZ are shown in dark green, while TetR-like repressor- and its putative cognate transporter-encoding genes are shown in blue and light blue, respectively. (B) Scheme of hybrid repressor construction. HTH - helix-turn-helix DNA binding domain; REC - putative ligand binding receiver domain.
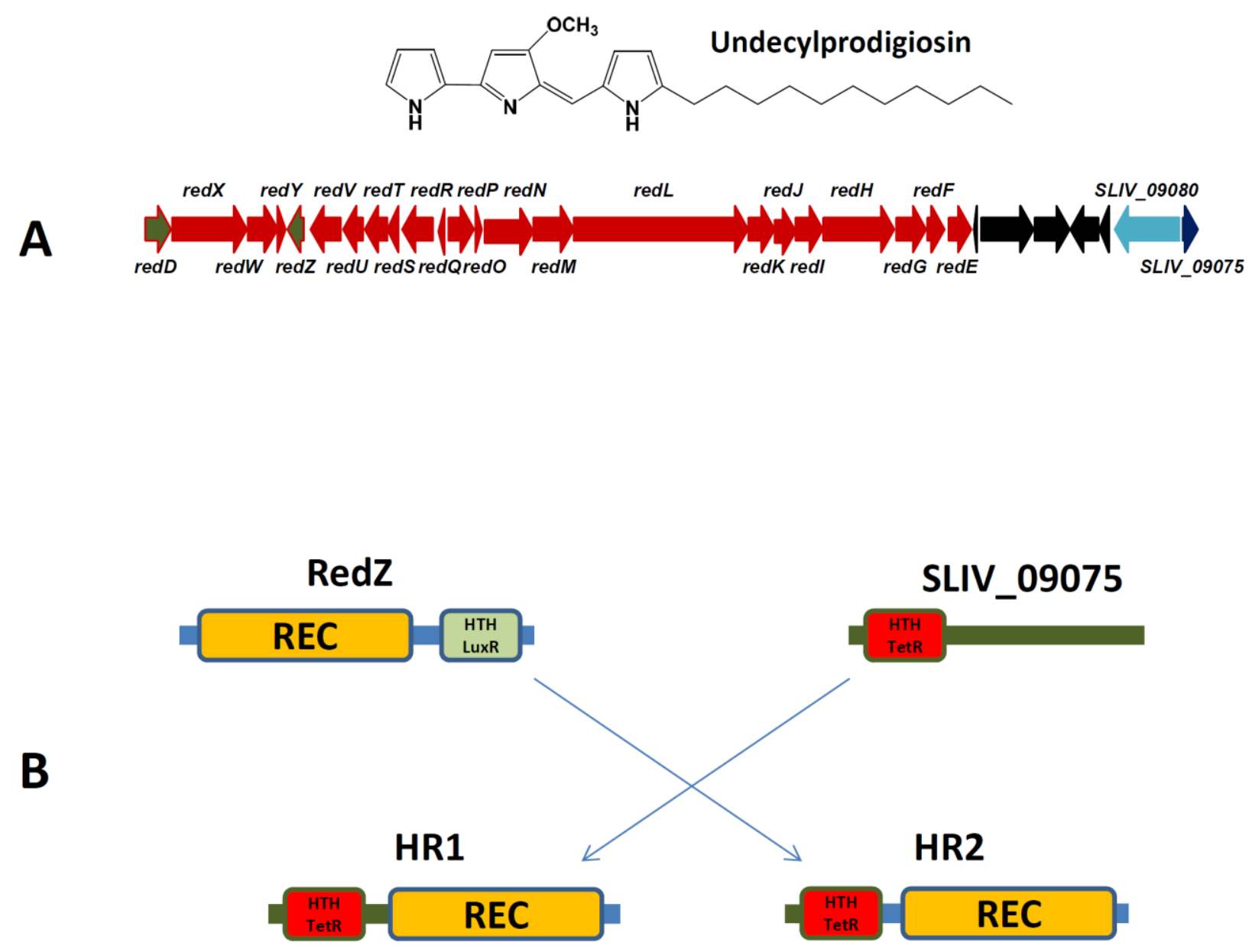
Figure 2. Organization of the coelimycin biosynthetic gene cluster and its parts used for the biosensor construction. Genes encoding putative positive activators are shown in orange, and a TetR-like repressor-encoding gene is shown in red. To construct a reporter, indigoidin synthetase gene bpsA was cloned downstream of the putative promoter/operator region controlling expression of type II thioesterase ScoT.

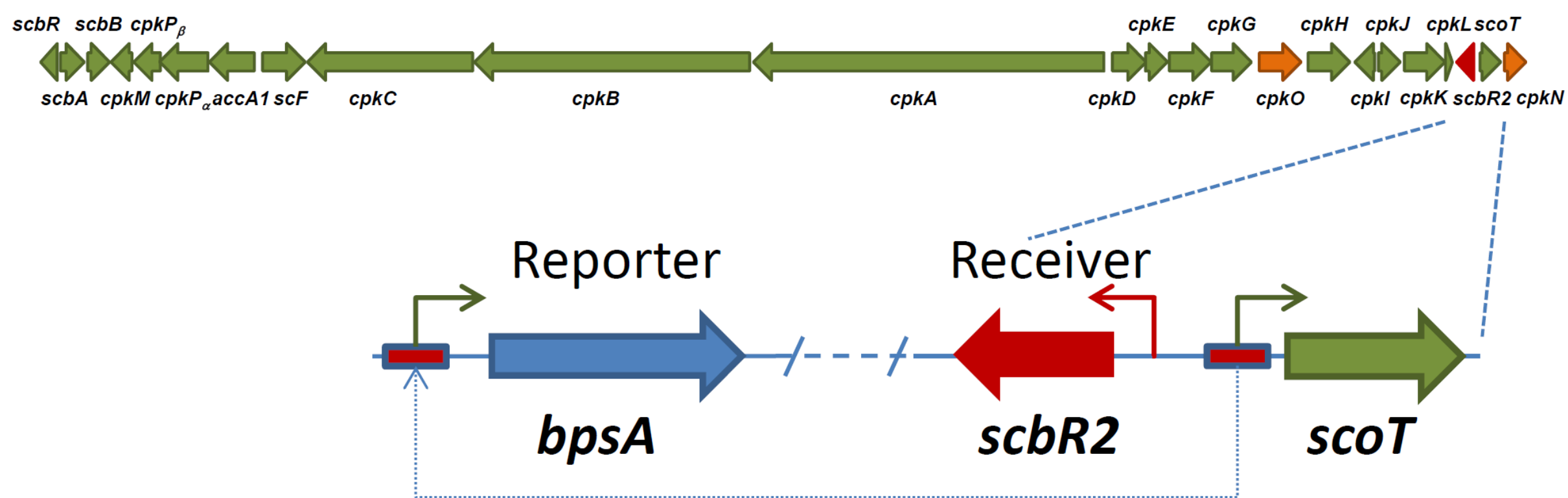


Figure 3. UV chromatogram (at $360 \mathrm{~nm}$ ) of metabolites produced by S. lividans RedStrep1 [pYQS040] and RedStrep1 [pYQS040/pYQS060] when grown in SG medium. The peak presumably corresponding to coelimycin P1 is indicated by arrow. More detailed analysis, including MS/MSbased identification of coelimycin P1, was done as reflected in Figure S2 (Supporting Information).

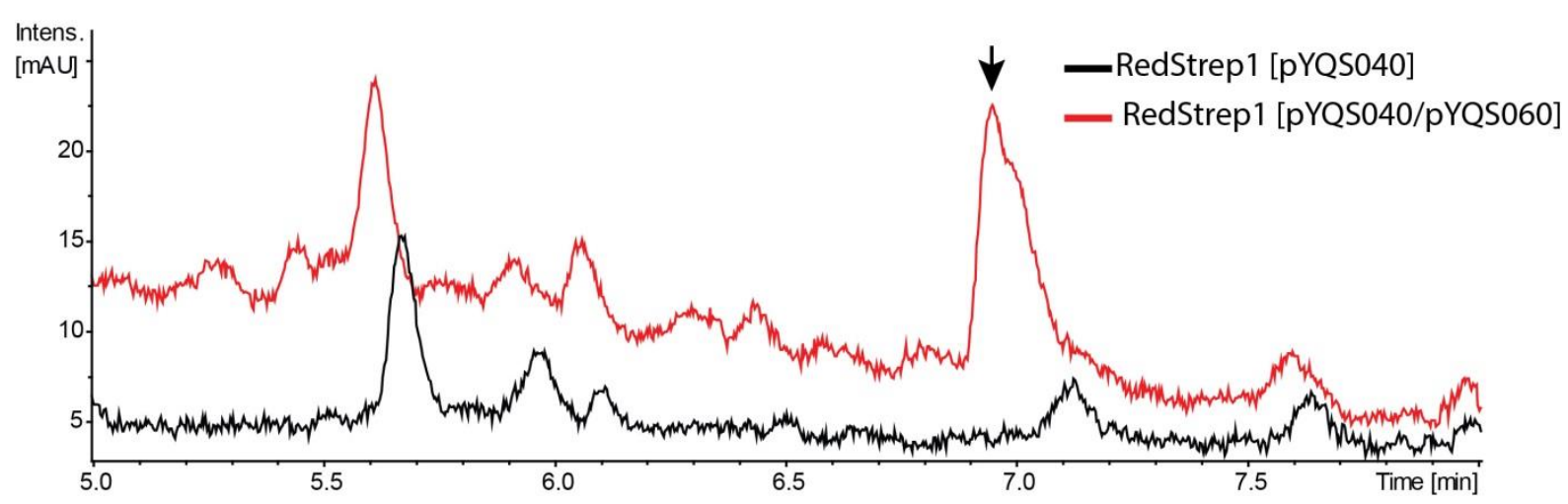

